\title{
Mechanistic Investigation into Phenol Oxidation by IBX Elucidated by DFT Calculations
}

\author{
Amritpal Kaur, Alireza Ariafard* \\ School of Natural Sciences (Chemistry), University of Tasmania, Private Bag 75, Hobart, TAS \\ 7001, Australia
}

\begin{abstract}
Density functional theory (DFT) at the SMD/M06-2X/def2-TZVP//SMD/M062X/LANL2DZ(d),6-31G(d) level was used to explore the regioselective double oxidation of phenols by a hypervalent iodine $(\mathrm{V})$ reagent (IBX) to give o-quinones. The oxidative dearomatization commences with the ligand exchange between IBX and phenol, yielding a phenolate complex, followed by the first redox process, which reduces iodine(V) to iodine(III). Both the processes (the ligand exchange and the first redox reaction) were found to be mediated by a less stable isomer of iodine $(\mathrm{V})$ species. We found that although the first redox process preferentially proceeds via an associative pathway, an electron withdrawing substituent on the phenol ring decreases its accessibility. The inspection of the electronic structure of the redox transition state indicates that the phenolate involved in the iodine(V) reduction has some phenoxenium character. The intrinsic stability of a phenoxenium ion is calculated to be highly sensitive to the substituent on the phenol ring. Since the electron withdrawing substituents considerably decrease the stability of the phenoxenium, they render the iodine(V) to iodine(III) reduction energy consuming. Once the first redox step has completed, a catechol-iodine(III)
\end{abstract}


complex is formed, from which the second redox process produces the final $o$-quinone product via a carboxylate-assisted transition structure. This transition structure gains stability by hydrogen bond interaction between the catechol $\mathrm{OH}$ and carboxylate group. Such an interaction results in the phenolate not having any phenoxenium character in the transition structure, thus making the activation barrier to the second redox step independent from the substituent on the phenol ring.

\section{Introduction}

Hypervalent iodine reagents provide a new approach for the oxidation of organic compounds in an eco-friendly manner as an alternative to transition metal catalysts, which are usually expensive and toxic to the environment. ${ }^{1}$ These kinds of reagents have been approved to serve as mild oxidants to produce many useful compounds; for example, they are well reported as useful compounds in dearomatizing phenols via oxidative processes. ${ }^{2}$

Interestingly, the nature of the product obtained from oxidation of phenols depends on the employed iodane. Reaction of phenols with hypervalent iodine(III) reagent PIDA [PIDA = phenyliodine(III) diacetate] in the presence of a nucleophile $(\mathrm{Nu}-\mathrm{H})$ gives cyclohexadienone II (Scheme 1a), ${ }^{2 a}$ whereas phenols react with hypervalent iodine(V) IBX reagent $(I B X=2$ iodobenzoic acid) and produce $o$-quinone $\mathbf{V}$ as the final product via a double oxidation process (Scheme 1b). ${ }^{3,4}$ 


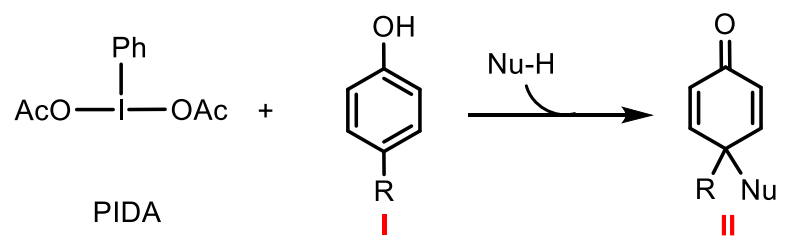

(a)

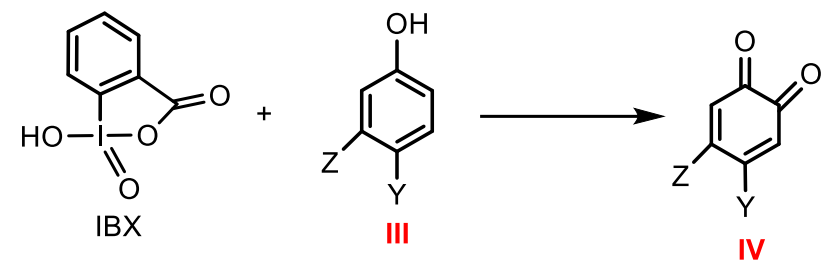

(b)

Scheme 1 Different products obtained from phenol oxidation by (a) PIDA and (b) IBX

The oxidative dearomatization of phenols mediated with PIDA was postulated to proceed via two different pathways, (i) associative and (ii) dissociative (Scheme 2). On that basis, a ligand exchange between phenol and PIDA should take place for the oxidative reaction to be initiated. The ensuing $\lambda^{3}$-iodane intermediate $\mathbf{V}$ was proposed as a starting point for both associative and dissociative mechanisms. In associative pathway, nucleophile $\mathrm{Nu}-\mathrm{H}$ attacks the phenolate ring of V, serving as a promoter for the phenol oxidation, which directly yields cyclohexadienone product II. By contrast, dissociative mechanism was assumed to proceed through fragmentation of $\lambda^{3}$ iodane $\mathbf{V}$ into $\mathrm{AcO}^{-}+\mathrm{PhI}+$ phenoxenium ion VII predisposed by an internal redox process. Finally, the formed phenoxenium is trapped by $\mathrm{Nu}-\mathrm{H}$ to generate product II. 


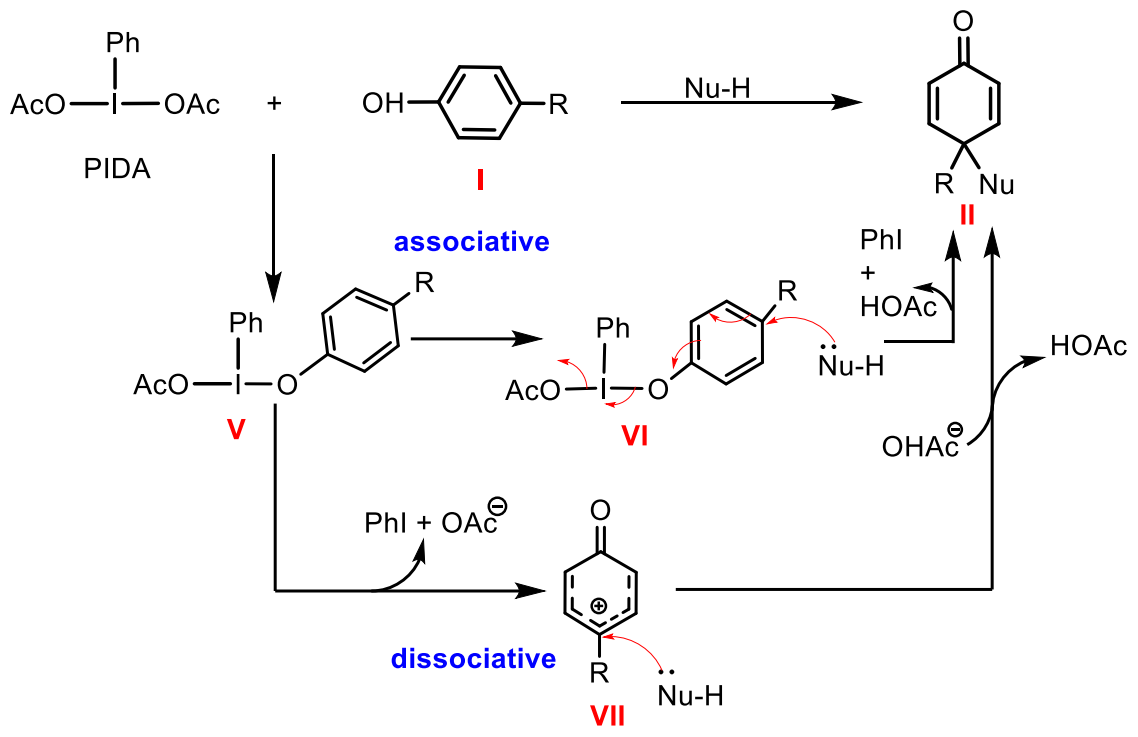

Scheme 2 Proposed conventional mechanism for phenol oxidation by PIDA via two associative and dissociative pathways.

In a recent publication, we modified the mechanism relating to PIDA-mediated phenol oxidation with the aid of density functional theory (DFT) calculations, the results of which are seen in Scheme $3 .^{5}$ We found that for phenol oxidation to proceed through a lower activation energy, the phenolate ligand requires to be initially dearomatized. Accordingly, the ligand exchange process between PIDA and phenol should occur via transition structure TSPIDA-VIII, resulting in species VIII, which is a branching point for both associative and dissociative pathways. In the associative pathway, the OAc ligand serves as a directing group for addition of $\mathrm{MeOH}$ to the dearomatized phenolate, which subsequently yields product II via transition structure TSvIII-II. In the dissociative pathway, intermediate VIII dissociates the OAc ligand assisted by HOAc to form intermediate IX, which is highly reactive towards redox process. The oxidation of phenolate in IX gives phenoxenium VII, the reaction of which with $\mathrm{MeOH}$ eventually generates product II. 


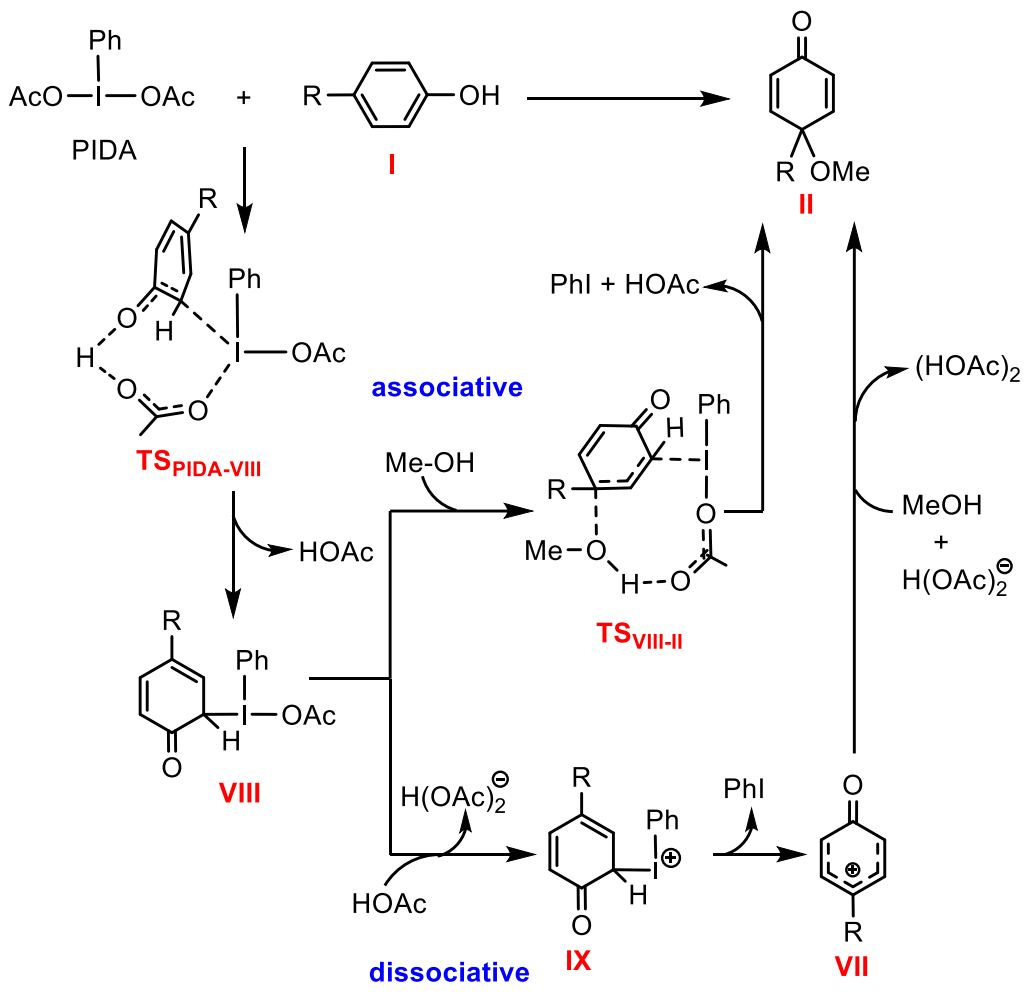

Scheme 3 Mechanism modified by DFT calculations at the M06-2X level of theory for phenol oxidation by PIDA via associative and dissociative pathways where $\mathrm{MeCN}$ was used as the solvent with $\mathrm{R}=\mathrm{Me}$ (for details, see ref. 5).

Inspired by the conventional mechanism given in Scheme 2, Pettus et al. proposed the mechanism put forward in Scheme 4 for double oxidation of phenols by IBX. ${ }^{3}$ Accordingly, the reaction was assumed to commence with a ligand exchange reaction, followed by reduction of iodine(V) species XI by intramolecularly delivering of an oxygen atom from the iodine to the least congested ortho carbon of the phenol. Subsequently, the iodine(III) intermediate XI tautomerizes to yield intermediate XII, which then the desired product IV is formed via oxidation of the aromatized phenolate by the iodine(III) center. 


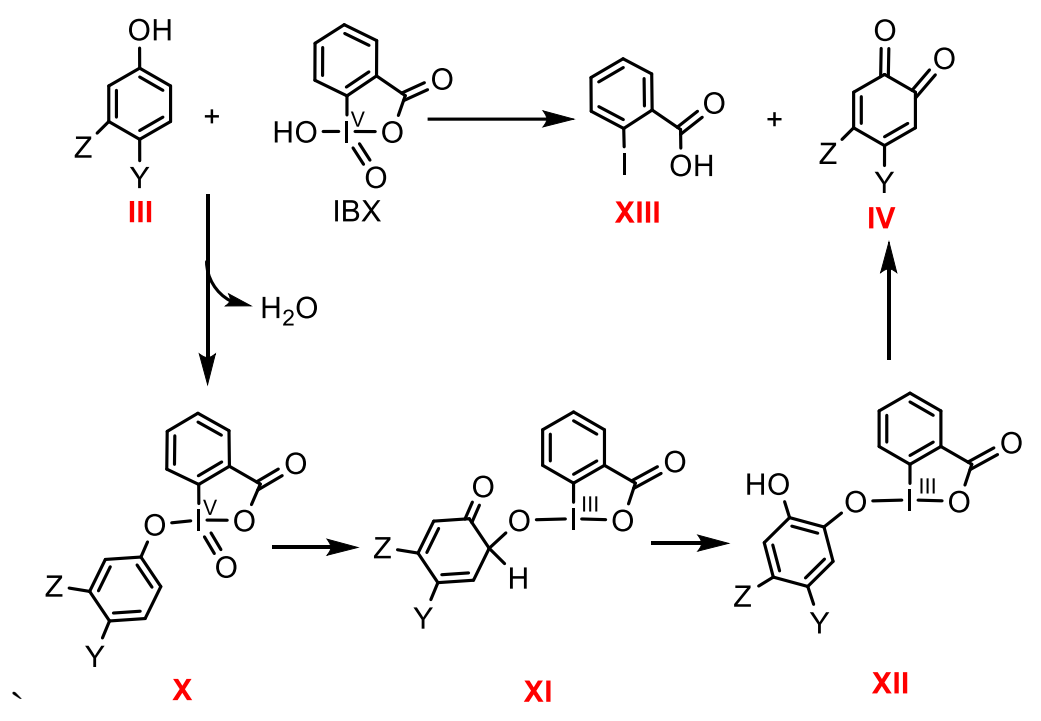

Scheme 4 Mechanism proposed by Pettus et al. (ref. 3) for double oxidation of phenols by IBX.

In this contribution, we intend to investigate the oxidation mechanism of phenols by iodine(V) reagent IBX with the aid of the DFT calculations at the M06-2X level of theory. ${ }^{6}$ As mentioned above, for phenol oxidation to occur by PIDA, phenolate is required to be dearomatized in order for the redox process to take place with more accessible activation energy. Now the question is whether a similar phenolate dearomatization is also needed for phenol oxidation by IBX. Additionally, although the ligand exchange between phenol and IBX has been proposed as an essential step for the oxidative dearomatization, its reaction mechanism is not very clear. This work exploits DFT-based calculations to provide a convincing mechanism for this key step.

Pettus et al. demonstrated that the phenol oxidation is not mediated by IBX if the phenol is substituted by an electron withdrawing group such as $\mathrm{C}(\mathrm{O}) \mathrm{R}$ and $\mathrm{NO}_{2} \cdot{ }^{3,7}$ Our computational results will provide a rationale behind this observation. We will show that the activation barrier to 
the reduction of iodine(V) to iodine(III) is significantly dependent on the phenol substituent nature while that of iodine(III) to iodine(I) is completely independent.

\section{Results and discussion}

As reported in the literature, structure A (Scheme 5) is the most stable isomer of IBX in which the oxo ligand is trans to the vacant site. ${ }^{8}$ Other less stable isomers $\mathbf{B}$ and $\mathbf{C}$ are likely to be formed in situ during the course of a reaction. In isomer $\mathbf{B}$, the oxo group is trans to the carboxylate while in isomer $\mathbf{C}$, it is trans to the hydroxide ligand (Scheme 5). We commenced our study by investigating which isomer (A, B, or $\mathbf{C})$ is responsible in driving the ligand exchange reaction.
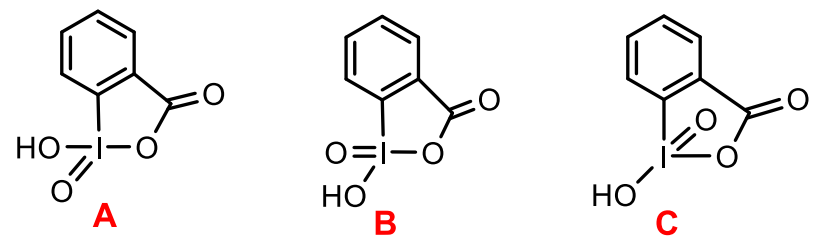

Scheme 5 Different isomers for IBX where $\mathbf{A}$ is the most stable form.

Ligand exchange between phenol and isomer A. Fig. 1 shows the calculated mechanism for ligand exchange between phenol 16-p-OMe and the most stable isomer A. For the ligand exchange to take place, the phenol is required to coordinate to IBX. The coordination gives species $\mathbf{1 7}$ with a relative free energy of $0.6 \mathrm{kcal} / \mathrm{mol}$. The proton transfer from the coordinated phenol to the hydroxide ligand leads to the completion of the ligand exchange. Such a proton transfer, which is assisted by a water molecule via transition structure TS17-18 is found to have an activation free energy of $20.1 \mathrm{kcal} / \mathrm{mol}$. It is worth noting that we were unable to locate any transition structure for the proton transfer without the assistance of a water molecule. The ligand exchange process via this variant by releasing two water molecules gives 19 having a relative free energy as high as $21.0 \mathrm{kcal} / \mathrm{mol}$. The trans positioning of two relatively strong $\sigma$-donor ligands (oxo and phenolate) 
in 19 causes formation of such an intermediate to be highly energetic, implying that the most stable isomer $\mathbf{A}$ is less likely to be involved in the ligand exchange process.

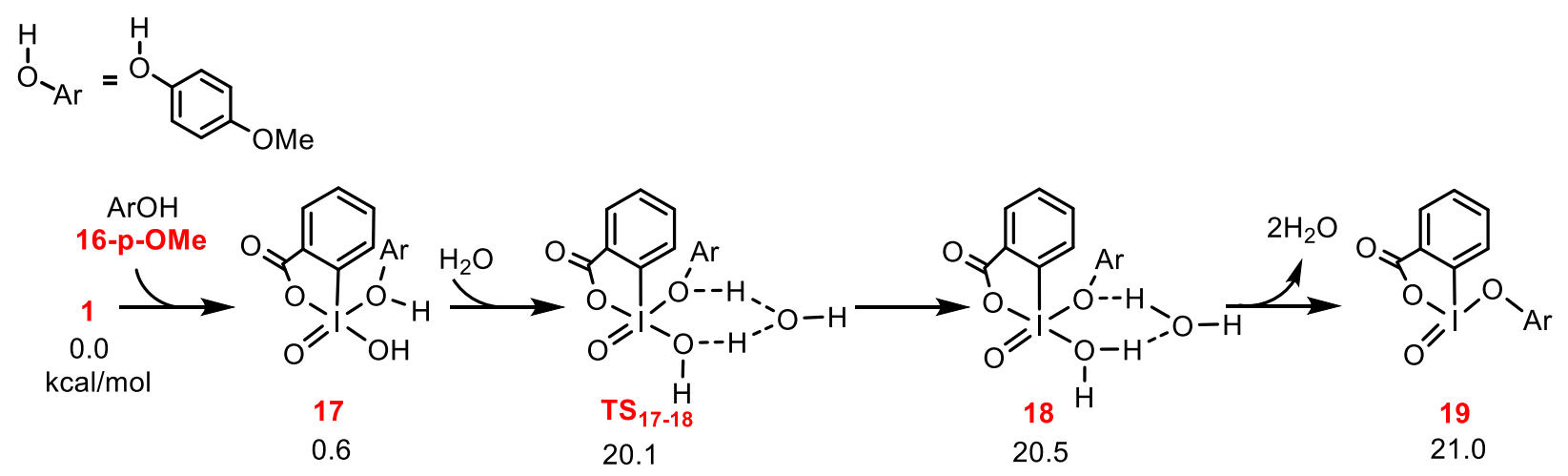

Fig. 1 Mechanism calculated for ligand exchange between phenol 16-p-OMe and the most stable isomer of IBX (A). The relative free energies are given in $\mathrm{kcal} / \mathrm{mol}$.

Ligand exchange between phenol and isomer B. The DFT calculation results for the ligand exchange between phenol and isomer $\mathbf{B}$ is shown in Fig 2. It finds that for the ligand exchange to be mediated by isomer $\mathbf{B}$, adduct $\mathbf{1 7}$ should participate in a proton transfer process involving migration of the hydroxide proton to the oxo ligand (Fig 2a). This proton transfer is computed to be assisted by a water molecule and occurs by transition structure $\mathbf{T S}_{17-22}$ with $\Delta \mathrm{G}^{\ddagger}=10.7$ $\mathrm{kcal} / \mathrm{mol}$. Once the adduct $\mathbf{2 2}$ has formed, it becomes a branching point for two competitive pathways ( $a$ and $b$, Fig. 2b). In pathway $a$, the $\mathrm{OH}$ proton of coordinated phenol is transferred to the carboxylate ligand via $\mathbf{T} \mathbf{S}_{\mathbf{2 2} 2 \mathbf{2 8}}$, followed by another proton transfer via $\mathbf{T} \mathbf{S}_{\mathbf{2 8}-24}$ to form $\mathbf{2 4}$. In pathway $b$, the $\mathrm{OH}$ proton of coordinated phenol is transferred to the oxo ligand via $\mathbf{T S}_{\mathbf{2 2} 2 \mathbf{2 3}}$, followed by another proton transfer via $\mathbf{T S}_{\mathbf{2 3 - 2 4}}$ to form $\mathbf{2 4}$. The activation barriers for these two pathways are comparable and much lower in energy than that calculated for the pathway illustrated in Fig. 1. This result suggests that isomer $\mathbf{B}$ is much more reactive than the more stable isomer $\mathbf{A}$ 
toward the ligand exchange. According to our calculations, a proton shuttling agent like water is essential for the corresponding proton transfers to occur with lower activation energies. We have to note that transition structures $\mathbf{T} \mathbf{S}_{22-23}, \mathbf{T S} \mathbf{S}_{23-24}, \mathbf{T} \mathbf{S}_{22-28}$ and $\mathbf{T} \mathbf{S}_{28-24}$, without the involvement of the water proton shuttling agent all are energetically higher with the relative free energies of 17.9, $23.1,13.2$, and $14.4 \mathrm{kcal} / \mathrm{mol}$, respectively.
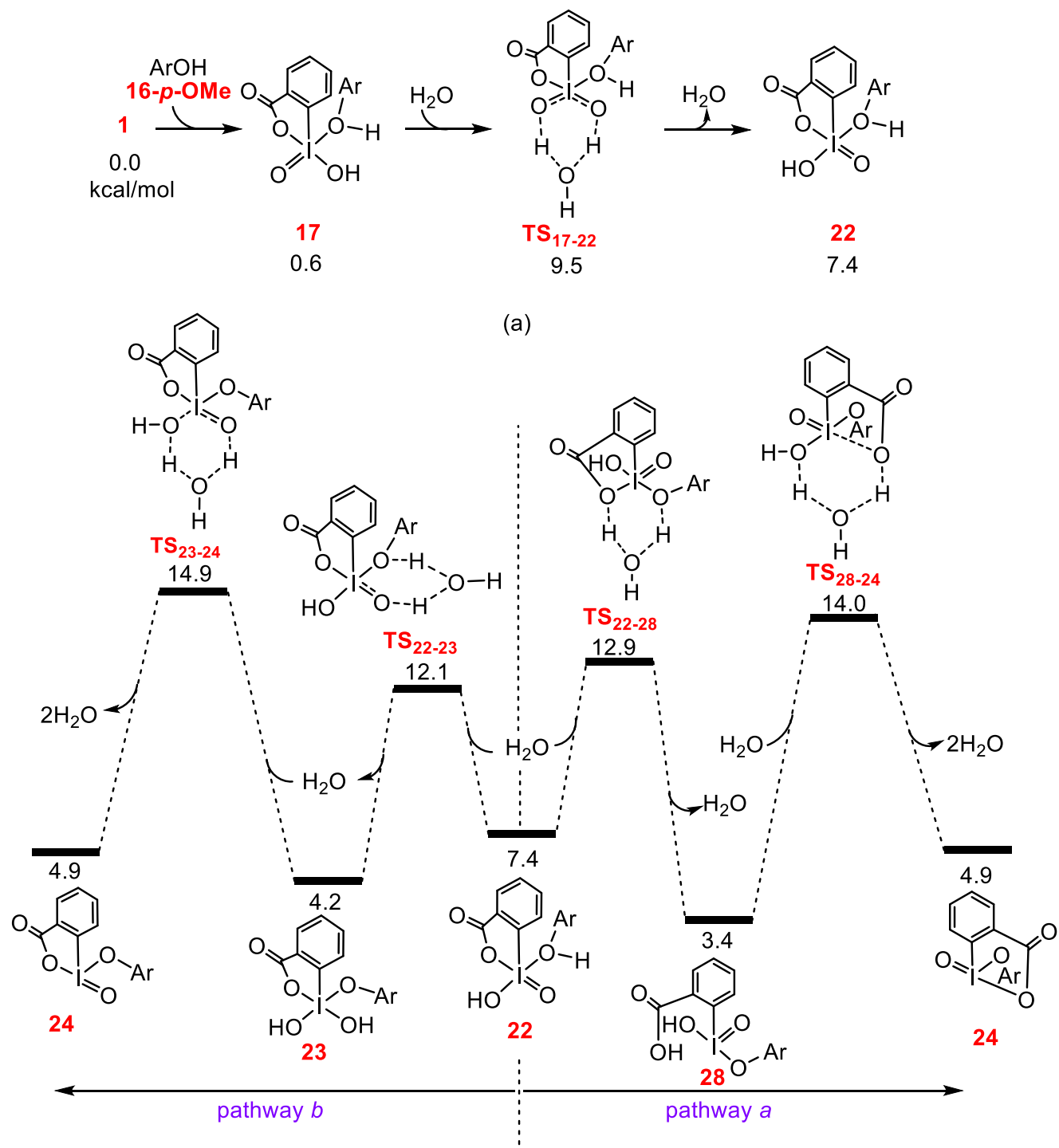

(b)

Fig. 2 (a) Mechanism calculated for formation of adduct 22 in which the phenol coordinates to isomer B. (b) Free energy profile for the ligand exchange starting from intermediate 22 via two pathways $a$ and $b$. The relative free energies are given in $\mathrm{kcal} / \mathrm{mol}$. 
Ligand exchange between phenol and isomer $\mathbf{C}$. Finally, the reactivity of isomer $\mathbf{C}$ toward the ligand exchange was investigated (Fig. 3). Two key transition structures ( $\mathbf{T S}_{\mathbf{2 0}}$ and $\mathbf{T S}_{\mathbf{2 1}}$ ) for this variant were located. Both transition structures $\mathbf{T S}_{\mathbf{2 0}}$ and $\mathbf{T} \mathbf{S}_{\mathbf{2 1}}$ with relative free energies of 28.2 $\mathrm{kcal} / \mathrm{mol}$ and $28.9 \mathrm{kcal} / \mathrm{mol}$, respectively, lie much higher in energy than transition structures $\mathbf{T} \mathbf{S}_{22}$ 23, $\mathbf{T S}_{23-24}$, $\mathbf{T S} 22-28$ and $\mathbf{T S} 28-24$ (Fig. 2b). Accordingly, any possibility by which isomer $\mathbf{C}$ is responsible to trigger the ligand exchange process is ruled out. The intrinsic instability of these two transition structures can be associated with the trans positioning of two relatively strong $\sigma$ donating oxo and hydroxide ligands. Comparison of the above results allows us to conclude that isomer $\mathbf{B}$ is far more reactive than isomers $\mathbf{A}$ and $\mathbf{C}$ in driving the ligand exchange reaction. ${ }^{9}$

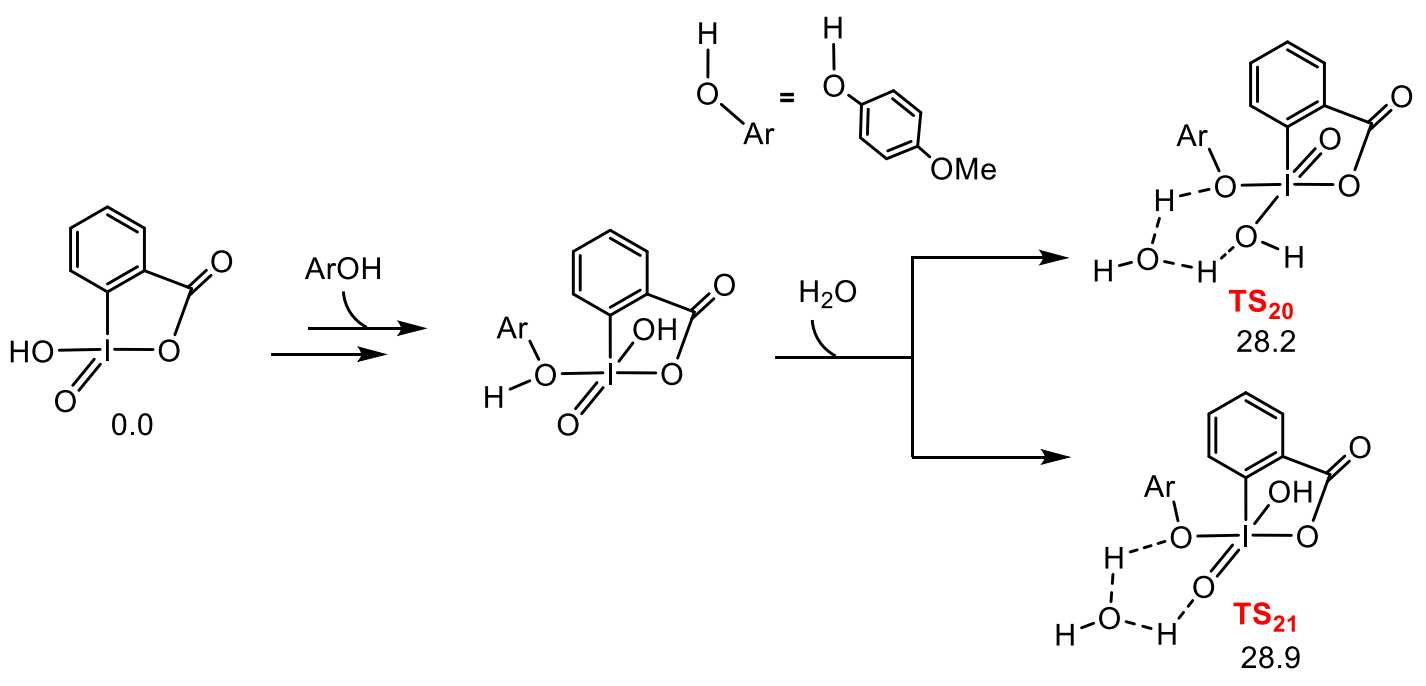

Fig. 3 Calculated vital transition structures for ligand exchange between phenol and isomer $\mathbf{C}$. The relative free energies are given in $\mathrm{kcal} / \mathrm{mol}$.

First redox process. As shown above (Fig. 2b), species $\mathbf{2 4}$ is the initial product of the ligand exchange process. The formed intermediate $\mathbf{2 4}$ is a bifurcation point for two different processes: (i) direct redox reaction and (ii) isomerization to $\mathbf{2 5}$ followed by redox reaction (Fig. 4). Three different mechanisms were found for the redox step: (a) associative, (b) dissociative and (c) single 
electron transfer (SET). In associative pathway, while iodine(V) is being reduced to iodine(III),

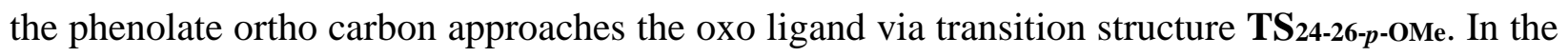
dissociative mechanism, two electrons from the phenolate are directly transferred to the iodine $(\mathrm{V})$ center achieved by lengthening of the I-O(phenolate) bond via transition structure $\mathbf{T S}_{\mathbf{3 2}}$. This transition structure is expected to form directly a phenoxenium ion but the high reactivity of such a carbocation does not allow it to be located as a minimum and results in formation of dearomatized phenolate complex $\mathbf{2 4}$. Finally, the SET mechanism involves the promotion of an electron from phenolate to the iodine $(\mathrm{V})$ center by passing through the minimum energy crossing point MECP1 and thus generates triplet adduct $\mathbf{3 3}$ with the relative free energy of $3.9 \mathrm{kcal} / \mathrm{mol}$. An inspection of the results presented in Fig. 4 implies that the associative pathway is more favorable than the dissociative and SET mechanisms by 3.2 and $5.2 \mathrm{kcal} / \mathrm{mol}$, respectively, suggesting that the iodine $(\mathrm{V})$ reduction by the phenolate starting from $\mathbf{2 4}$ most likely proceeds via the associative mechanism. The favorability of the associative mechanism over the other variants explains the regioselectivity experimentally observed for the IBX-mediated oxidation of phenols. ${ }^{3}$

As mentioned above, $\mathbf{2 4}$ has the potential to isomerize to a more stable form (25) from which the redox reaction occurs. The computed activation energy for the redox reaction to proceed from $\mathbf{2 5}$ via $\mathbf{T S}_{25}$ is $24.2 \mathrm{kcal} / \mathrm{mol}$, which is comparably higher than the direct reduction from $\mathbf{2 4}$. This result indicates that the more stable isomer $\mathbf{2 5}$ is much more reactive than the less stable isomer $\mathbf{2 4}$ toward the redox process. The transition structure for isomerization $\left(\mathbf{T S}_{\mathbf{2 4 - 2 5}}\right)$ is also calculated to lie higher

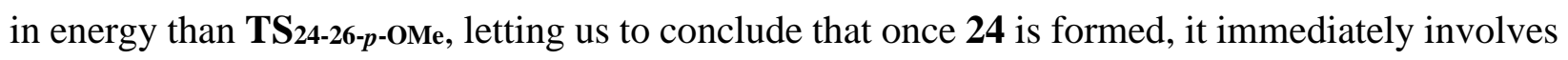
in the redox process. In other words, the more stable isomer $\mathbf{2 5}$ is less likely to be formed during this oxidative dearomatization process and when the ligand exchange reaction produces $\mathbf{2 4}$, it directly participates in the iodine $(\mathrm{V})$ reduction via the associative mechanism. 
The higher reactivity of $\mathbf{2 4}$ versus $\mathbf{2 5}$ is consistent with the results of Goddard et al. who proposed that a hypervalent twist is required for the redox step of IBX-mediated alcohols oxidation. ${ }^{8 \mathrm{e}} \mathrm{We}$ found that the iodine $(\mathrm{V})$ reduction in $\mathbf{2 4}$ forces two electrons from the phenolate to a $2 \mathrm{c}-2 \mathrm{e} \sigma^{*}$ orbital while that in $\mathbf{2 5}$ causes a $3 \mathrm{c}-4 \mathrm{e} \sigma^{*}$ orbital to be occupied. Since a $2 \mathrm{c}-2 \mathrm{e} \sigma^{*}$ orbital lies much lower in energy than a $3 \mathrm{c}-4 \mathrm{e} \sigma^{*}$ orbital, $\mathbf{2 4}$ is more susceptible than $\mathbf{2 5}$ to drive the redox step (for more details see Fig S1). 

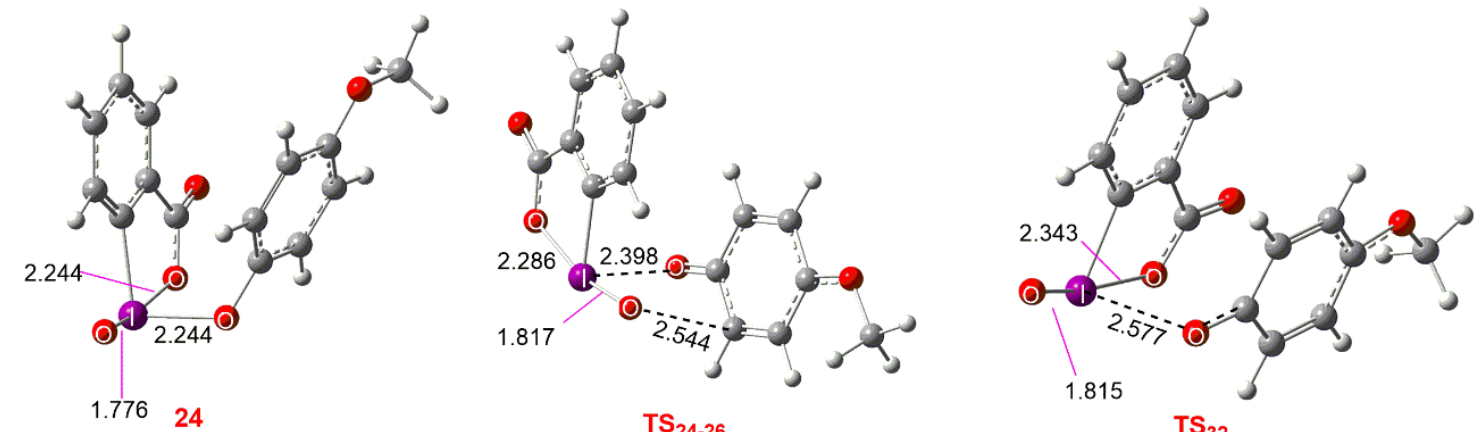

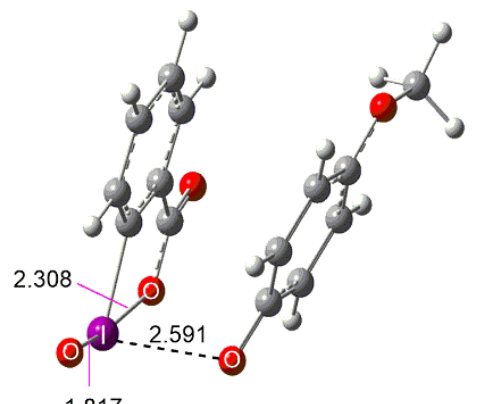

1.817

MECP1

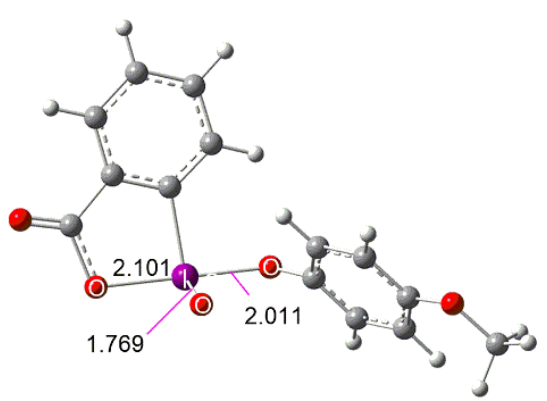

25

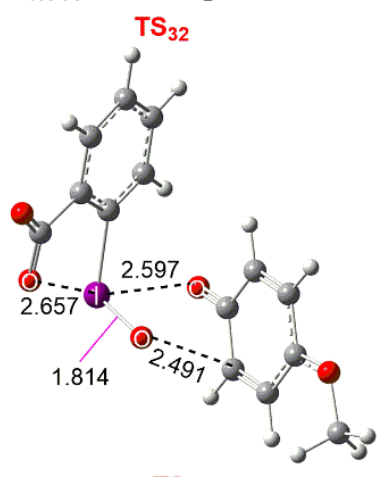

$\mathrm{TS}_{25}$<smiles>COC(=O)O[PH]1(O)Oc2cc(ccc2OC)C(=O)c2ccccc21</smiles><smiles>CC[14CH2][14CH2][14CH2]O[14CH2]O[14CH2]O[14CH]1C=CC(OC)=C1</smiles>

2द⿱2

redox from $\mathbf{2 5}$

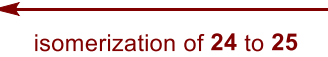

redox from 24

24

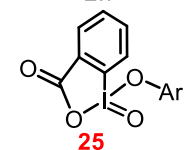

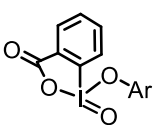
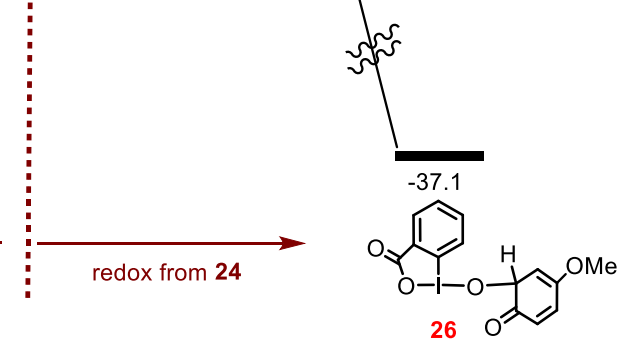
Fig. 4 Free energy profile calculated for redox reaction from 24 via three different pathways: associative, dissociative, and SET and isomerization of $\mathbf{2 4}$ to 25 followed by a redox process via transition structure $\mathbf{T S}_{\mathbf{2 5}}$. The relative free energies and bond distances in 3D structures are given in $\mathrm{kcal} / \mathrm{mol}$ and $\AA$, respectively.

\section{Can a dearomatized phenolate complex be a key intermediate for the oxidative} dearomatization reaction? As discussed in the Introduction, for phenol dearomatization by PIDA to proceed with a reasonable activation barrier, the phenolate ligand needs to be initially dearomatized. In a recent publication, ${ }^{5}$ we showed that the ligand exchange between PIDA and a phenol forms a dearomatized phenolate iodine complex via TSPIDA-vIII (Scheme 3). Now, the question is whether there is any possibility for IBX to form a dearomatized phenolate complex. As demonstrated above, the most reactive isomer of IBX toward the ligand exchange is species $\mathbf{B}$ (Fig. 2). Fig. 5 shows the free energy profile relating to formation of dearomatized phenolate complex 24' generated by the ligand exchange between phenol 16-p-OMe and IBX isomer $\mathbf{B}$. The $\pi$-complex $22^{\prime}$ with a relative free energy of $4.8 \mathrm{kcal} / \mathrm{mol}$ is surmised as the key intermediate. The formed $\pi$-complex can then undergo a proton transfer from the coordinated phenol to the oxo ligand via transition structure $\mathbf{T S}_{\mathbf{2 2}} \mathbf{2}^{-23^{\prime}}$ ' to give $\mathbf{2 3}^{\prime}$ with a free energy of $18.6 \mathrm{kcal} / \mathrm{mol}$. A second proton transfer assisted by water molecule subsequently takes place by passing through transition structure $\mathbf{T S}_{\mathbf{2 3}} \mathbf{2}^{-24^{\prime}}$, leading to completion of ligand exchange process and formation of dearomatized phenolate complex $\mathbf{2 4}^{\prime}$. The located transition structure $\mathbf{T} \mathbf{S}_{\mathbf{2 3}} \mathbf{\prime}^{\prime} \mathbf{2} \mathbf{4}^{\prime}$ with $\Delta \mathrm{G}^{\ddagger}=26.8$ $\mathrm{kcal} / \mathrm{mol}$ are much higher in energy than $\mathbf{T S} 22-23$, $\mathbf{T S} 23-24, \mathbf{T S} 22-28$ and $\mathbf{T S} 28-24$ (Fig. 2b), implying that the ligand exchange process is unlikely to yield a dearomatized phenolate complex. Indeed, the trans positioning of two relatively strong $\sigma$-donating ligands (dearomatized phenolate and hydroxide) in $\mathbf{2 3}^{\prime}$ results in this intermediate being highly energetic, rendering this alternative for ligand exchange unfavorable. ${ }^{10}$ 
Another variant for formation of $\mathbf{2 4}^{\prime}$ is that the aromatized $\mathbf{2 4}$ undergoes tautomerization via an energetically available transition structure. Attempts to find such a transition structure was unsuccessful and we found that the most accessible pathway for formation of $\mathbf{2 4}$ ' is the sequence

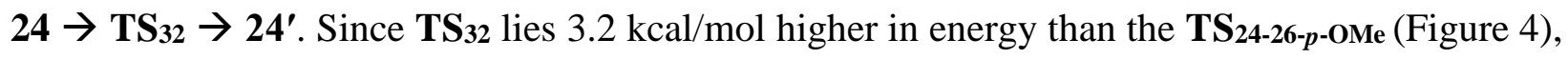
the redox reaction from $\mathbf{2 4}$ is anticipated to occur much faster than the tautomerization. The above findings allow us to propose that the dearomatized phenolate complex $\mathbf{2 4}$ ' is unlikely to be a key species for phenol oxidation by IBX.

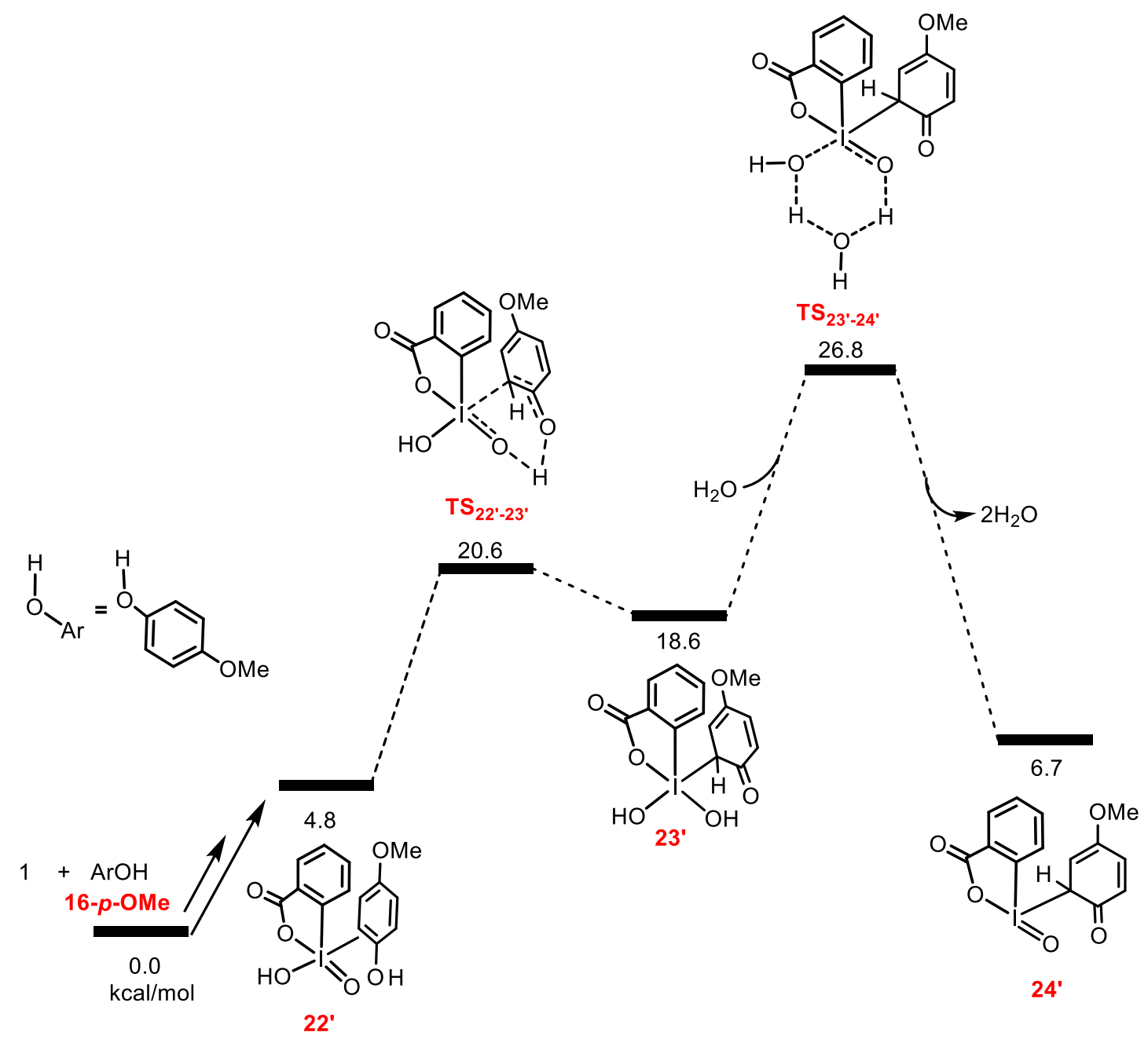

Fig. 5 Free energy profile for ligand exchange between IBX isomer $\mathbf{B}$ and phenol 16-p-OMe leading to dearomatized phenolate complex $\mathbf{2 4}^{\prime}$. The relative free energies are given in $\mathrm{kcal} / \mathrm{mol}$. 
Second redox process. Following the mechanism proposed by Pettus et al (Scheme 4), once intermediate 26 has produced, ${ }^{11}$ it undergoes tautomerization to give the iodine(III) intermediate 27-H,H,OMe,H from which another redox process occurs to form the $o$-quinone product 37 . This tautomerization is calculated to proceed with a free energy barrier of $17.3 \mathrm{kcal} / \mathrm{mol}$ in an exergonic fashion $\left(\Delta \mathrm{G}_{\mathrm{rxn}}=-19.8 \mathrm{kcal} / \mathrm{mol}\right)$ where a water cluster is used as a proton shuttling agent (Fig. 6).

Three different pathways $(c, d$ and $e$ ) are found for the reduction of iodine(III) center by the phenolate starting from intermediate 27-H,H,OMe,H (Fig. 7). The redox reaction in pathways $c$ proceeds by the simultaneous lengthening of both the I-O bonds via transition structure $\mathbf{T S}_{34}$. Due to the transfer of two electrons from phenolate to a high lying 3C-4e $\sigma^{*}$ orbital (Fig. S1), pathway $c$ is calculated to be very energy demanding with $\Delta \mathrm{G}^{\dagger}=31.3 \mathrm{kcal} / \mathrm{mol}$. Species $\mathbf{T S}_{35}$ is the key transition structure for pathway $d$ in which the carboxylate group is rotated by $90^{\circ}$ to allow the redox reaction occurring by transferring two electrons from the phenolate to a $2 \mathrm{C}-2 \mathrm{e} \sigma^{*}$ orbital which is much lower in energy than a $3 \mathrm{C}-4 \mathrm{e} \sigma^{*}$ orbital. This transition structure is still highly energetic with a free energy of $30.1 \mathrm{kcal} / \mathrm{mol}$ because it suffers from the cleavage of the strong I$\mathrm{O}$ (carboxylate) bond. Pathway $e$ is similar to pathway $d$ with the difference that the corresponding

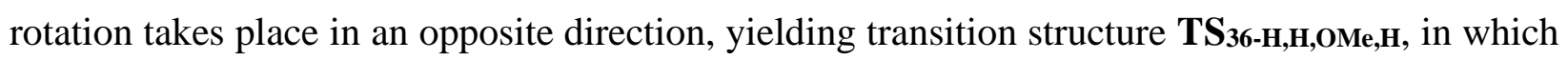
the anionic carboxylate is stabilized by a hydrogen bond interaction with the $\mathrm{OH}$ group on the

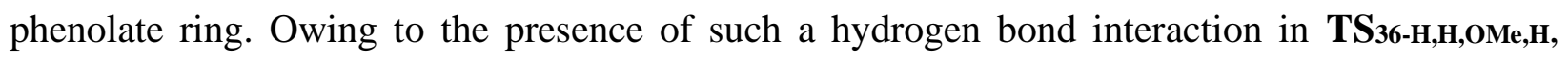
pathway $e$ with $\Delta \mathrm{G}^{\ddagger}=22.8$ is computed to be more favored than the other two pathways, a result which led us to conclude that the final redox step most likely proceeds via this carboxylate-assisted route. 


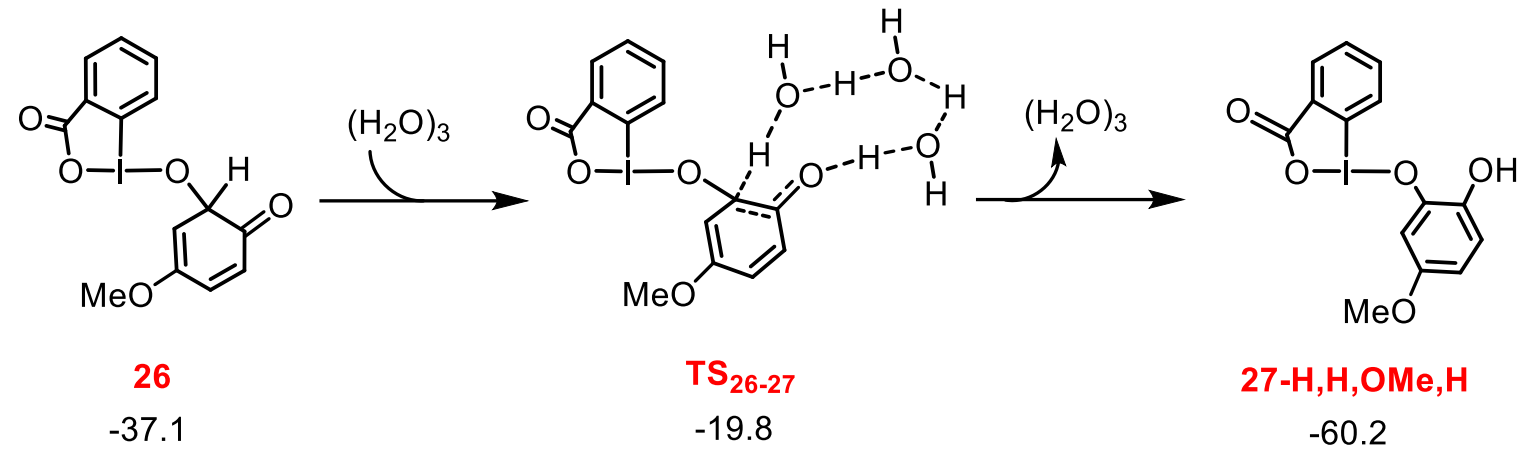

Fig. 6 Mechanism calculated for tautomerization $26 \rightarrow 27-\mathbf{H}, \mathbf{H}, \mathbf{O M e}, \mathrm{H}$ assisted by a water cluster. The relative free energies are given in $\mathrm{kcal} / \mathrm{mol}$.

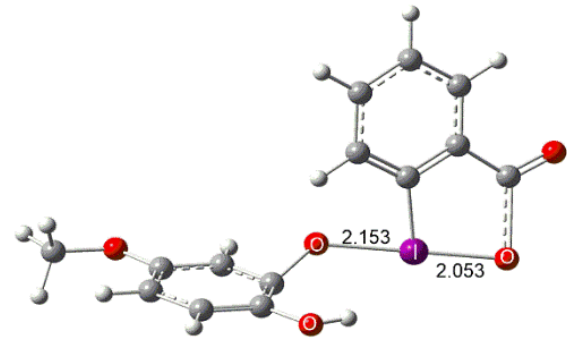

27
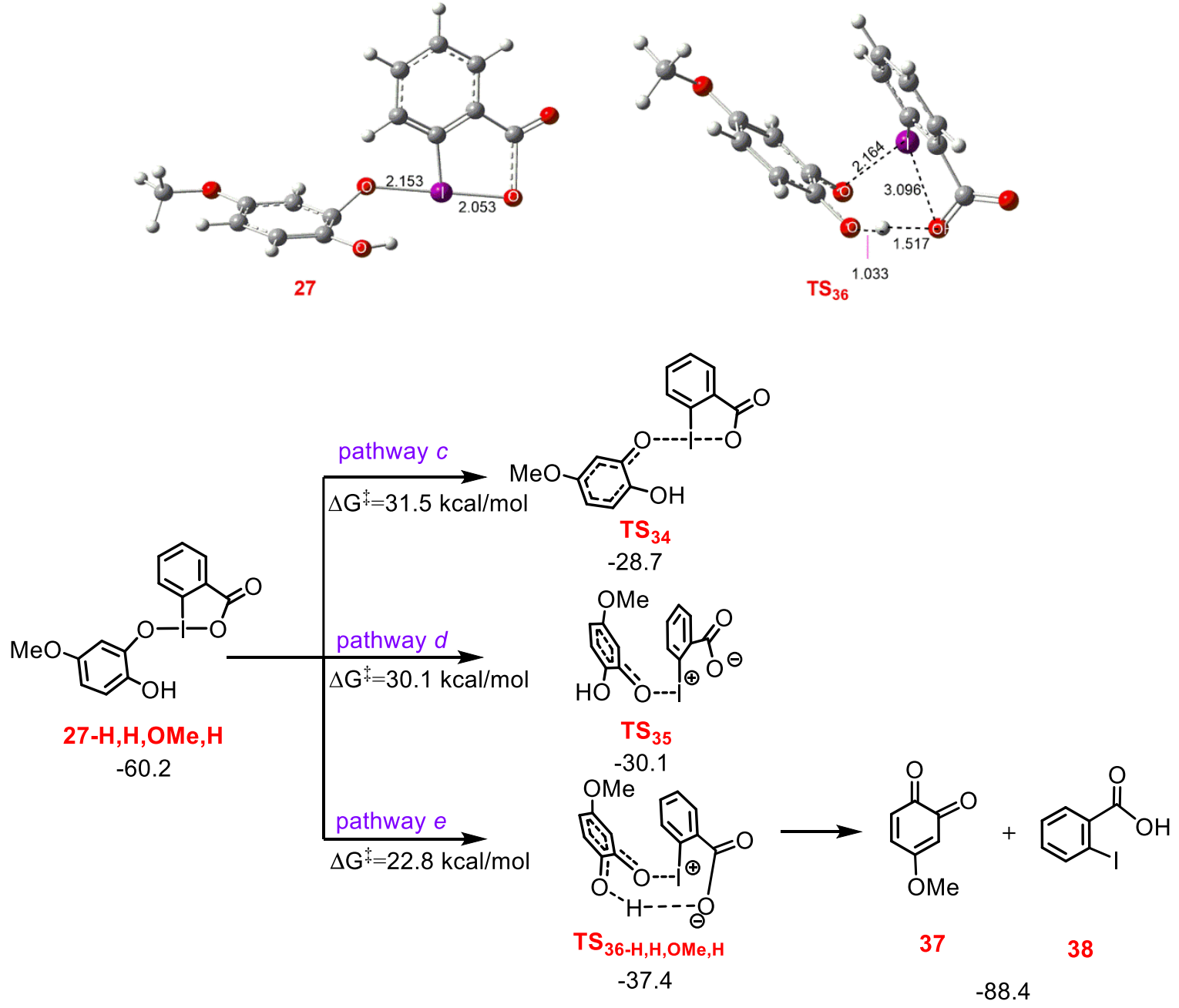

Fig. 7 Three different transition structures calculated for the second redox process. The relative free energies and bond distances in 3D structures are given in $\mathrm{kcal} / \mathrm{mol}$ and Å, respectively. 
Full free energy profile. Figure 8 summarizes our findings regarding oxidation of phenol 16-pOMe by IBX. Accordingly, the oxidation commences with coordination of the phenol to IBX followed by a proton transfer process via transition structure $\mathbf{T S}_{17-22}$ to generate $\mathbf{2 2}$. Our calculations showed that although $\mathbf{2 2}$ is an isomer for $\mathbf{1 7}$ with significantly lower stability, it is much more reactive toward the ligand exchange process and thus formation of key intermediate 24 takes place from this less stable isomer mainly via two transition structures $\mathbf{T S}_{\mathbf{2 2 - 2 8}}$ and $\mathbf{T} \mathbf{S}_{\mathbf{2 8 - 2 4}}$. The formed iodine $(\mathrm{V})$ intermediate $\mathbf{2 4}$ is a branching point for two routes. Either it isomerizes to a more stable structure $\mathbf{2 5}$, or it undergoes a redox process. The redox process from $\mathbf{2 4}$ is predicted

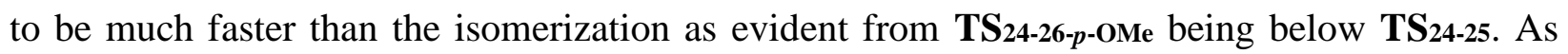
such, once $\mathbf{2 4}$ has formed, it directly involves the redox process to give $\mathbf{2 6}$ via associative transition structure TS24-26-p-OMe in which the oxo group is being coupled with phenolate ortho carbon while the $\mathrm{I}-\mathrm{O}$ (phenolate) bond is cleaved. Subsequently, a tautomerization process from $\mathbf{2 6}$ takes place and intermediate $\mathbf{2 7}-\mathbf{H}, \mathbf{H}, \mathbf{O M e}, \mathbf{H}$ is produced. The resultant intermediate then undergoes a final redox process via the carboxylate-assisted transition structure $\mathbf{T S}_{36-\mathbf{H}, \mathbf{H}, \mathbf{O M e}, \mathbf{H}}$ to give $o$-quinone product 37 . 


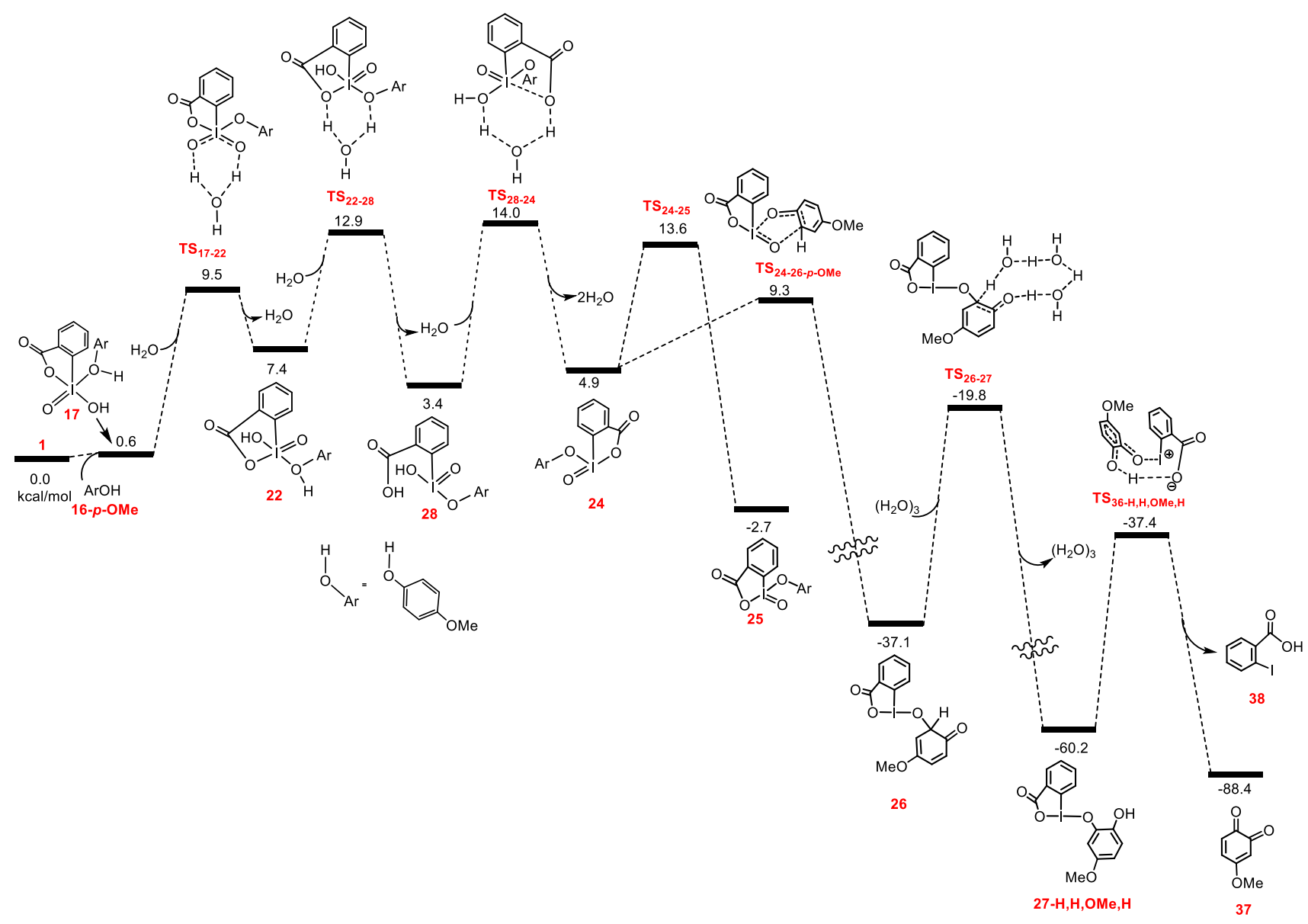

Fig. 8 A full free energy profile for double oxidation of phenol 16-p-OMe by IBX. The relative free energies are given in $\mathrm{kcal} / \mathrm{mol}$.

Effect of substituents on the first redox process. As discussed in the Introduction, Pettus et al. observed that when the phenol is substituted by the electron withdrawing groups such as $\mathrm{C}(\mathrm{O}) \mathrm{R}$ and $\mathrm{NO}_{2}$, the phenol oxidative dearomatization reaction is ceased. To rationalize this experimental finding, the activation free energy for the redox step $\left(\Delta \mathrm{G}^{\ddagger}\right)$ via transition structure $\mathbf{T} \mathbf{S}_{\mathbf{2 4 - 2 6}-\mathbf{x}}$ (eq 1$)$ for different substituents on the phenol ring was calculated. Several points are obvious from Table 1. First, consistent with the experimental observations, the electron withdrawing substituents such as $\mathrm{CN}, \mathrm{NO}_{2}$, and $\mathrm{C}(\mathrm{O}) \mathrm{CH}_{3}$ increase the redox activation free energy to a value greater than 26 
$\mathrm{kcal} / \mathrm{mol}$. Second, for an electron donating substituent like OMe, the activation free energy of the redox step is mainly affected by the position of the substituent. If the para position is substituted by $\mathrm{OMe}$, the activation barrier is reduced to $9.3 \mathrm{kcal} / \mathrm{mol}$ when it compares with $\mathrm{X}=\mathrm{H}$ having $\Delta \mathrm{G}^{\ddagger}$ $=23.7 \mathrm{kcal} / \mathrm{mol}$. The presence of the OMe group at the ortho position also causes the activation free energy to be reduced $\left(\Delta \mathrm{G}^{\ddagger}=19.5 \mathrm{kcal} / \mathrm{mol}\right)$, but not as much as that found for the para position. The meta positioning of OMe on the phenol ring has almost no significant influence on the redox activation barrier with $\Delta \mathrm{G}^{\ddagger}=24.5 \mathrm{kcal} / \mathrm{mol}$. Third, the replacement of the para hydrogen with chlorine does not significantly affect the redox activation barrier.

The $\Delta \mathrm{E}$ values (Table 1) for the isodesmic reaction outlined in eq 2 allow us to provide some insights into the relative stability of phenoxenium ions. Accordingly, the nature of the substituent was found to have a very strong effect on the stability of the phenoxenium as evidenced by the fact that the $\Delta \mathrm{E}$ values span a huge range from -32.9 to $21.3 \mathrm{kcal} / \mathrm{mol}$. Exactly the same trends are observed for $\Delta \mathrm{E}$ and $\Delta \mathrm{G}^{\ddagger}$. The electron withdrawing substituents especially at the para position decrease the stability of the phenoxenium. In contrast, the electron donating substituents at the ortho and para positions increase the stability of phenoxenium with a greater effect on the para position. The presence of an electron donating substituent on the meta position almost has no significant influence on the phenoxenium stability.

A strong correlation between the $\Delta \mathrm{E}$ and $\Delta \mathrm{G}^{\ddagger}$ values with $\mathrm{R}^{2}=0.92$ suggests that the activation free energy of the redox step is affected by the stability of the phenoxenium; the higher the stability of the phenoxenium, the lower the redox activation free energy. This result implies that although the reduction of iodine(V) to iodine(III) proceeds via an associative transition structure TS $_{24-26-}$ x), ${ }^{12}$ the phenolate ligand in this structure has some phenoxenium character which in turn affects the activation barrier to the redox step. The NPA charge analysis validates this supposition. For 
example, the charge on the whole phenolate ligand increases from -0.446 in 24 to +0.087 in $\mathbf{T S}_{\mathbf{2 4}}$ -

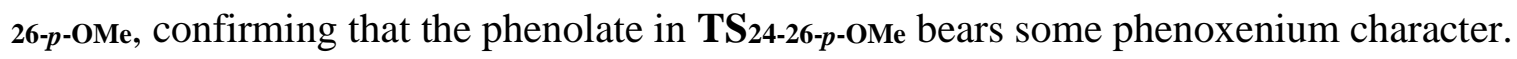

Table 1 Free energy change $\left(\Delta G^{\ddagger}\right)$ for transformation $\mathbf{1 6 - X} \rightarrow \mathbf{T S}$ 24-26-x (eq. 1) along with the potential energy change $(\Delta \mathrm{E})$ for the isodesmic reaction given in eq. 2 .<smiles>[X]c1cccc(O)c1</smiles>

$\mathrm{TS}_{24-26-\mathrm{X}}$<smiles>[X]c1ccc(O)cc1[X]</smiles>

\begin{tabular}{|c|c|c|}
\hline $\mathrm{X}$ & $\Delta \mathrm{G}^{\ddagger}$ & $\Delta \mathrm{E}$ \\
\hline$p$-OMe & 9.3 & -32.9 \\
\hline$p-\mathrm{Me}$ & 21.0 & -11.1 \\
\hline$p-\mathrm{Cl}$ & 23.9 & -1.4 \\
\hline $\mathrm{H}$ & 23.7 & 0.0 \\
\hline$p-\mathrm{C}(\mathrm{O}) \mathrm{CH} \mathrm{H}_{3}$ & 27.1 & 8.5 \\
\hline$p-\mathrm{CN}$ & 28.4 & 15.7 \\
\hline$p-\mathrm{NO} 2$ & 29.1 & 21.3 \\
\hline$o-\mathrm{OMe}$ & 19.5 & -19.0 \\
\hline
\end{tabular}




\begin{tabular}{|c|c|c|}
\hline$o-\mathrm{NO} 2$ & 26.4 & 14.5 \\
\hline$m-\mathrm{OMe}$ & 24.5 & 2.7 \\
\hline$m-\mathrm{NO}_{2}$ & 27.0 & 13.9 \\
\hline
\end{tabular}

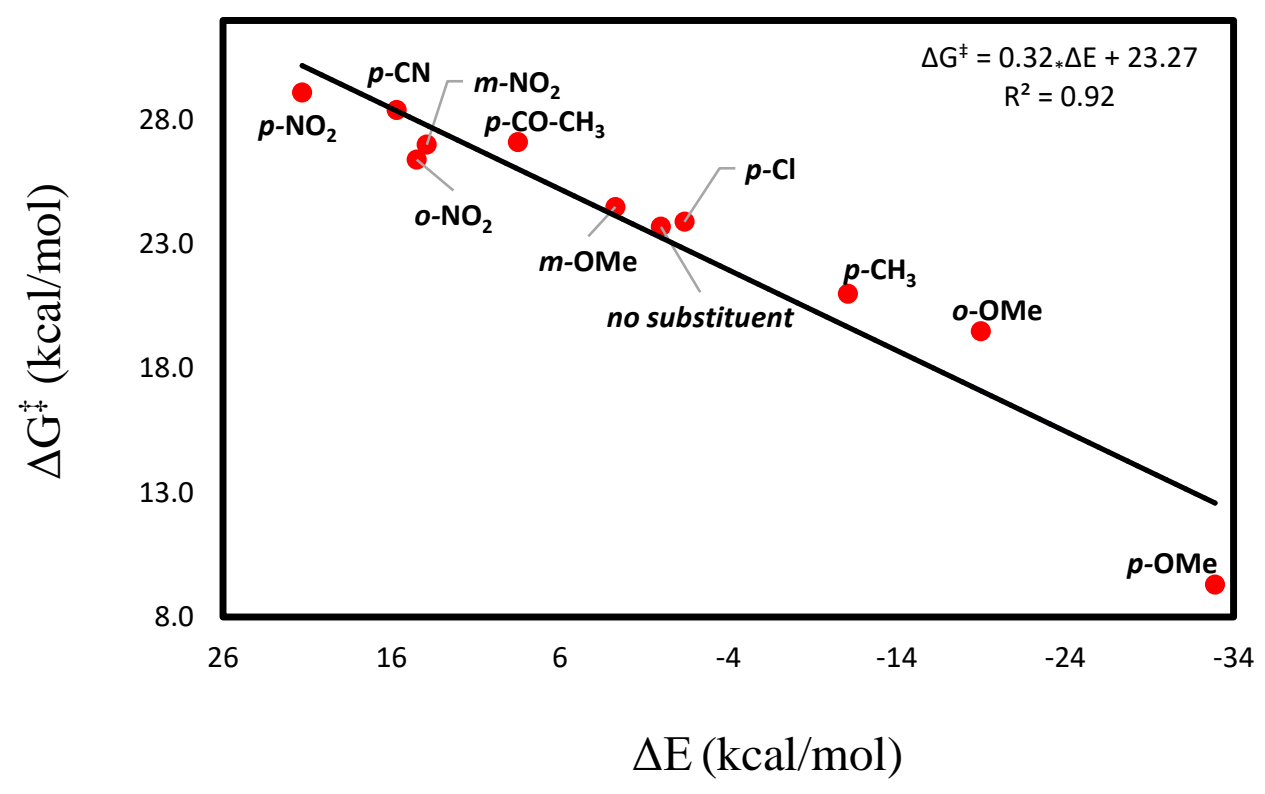

Fig. 9 A plot of correlation between $\Delta \mathrm{G}^{\ddagger}$ and $\Delta \mathrm{E}$ based on the data given in Table 1.

Effect of substituents on the second redox process. As revealed in Table 1, the activation barrier to the first redox reaction is strongly dependent on the substituent in the phenol ring. Now the question is whether a similar trend is present for the second redox process. To investigate this, we chose two substituents $\mathrm{OMe}$ and $\mathrm{CN}$ at different positions on the phenol ring of species 27$\mathbf{W}^{\prime}, \mathbf{X}^{\prime}, \mathbf{Y}^{\prime}, \mathbf{Z}^{\prime}$ as shown in Table 2. Interestingly, in contrast to the first redox process, the second

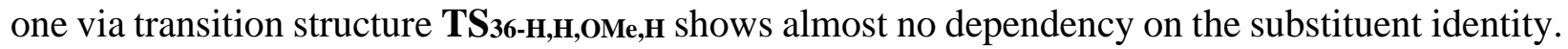
Indeed, although the isodesmic reaction given in Table 2 (eq 4) indicates that an electron withdrawing substituent decreases the stability of the phenoxenium, this trend is not reflected on 
the redox activation barrier. This contradicting result could be attributed to the fact that the

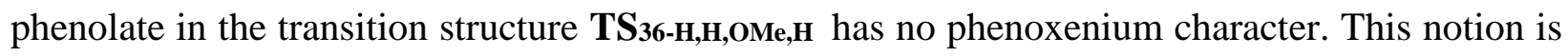
corroborated by the NPA charge analysis; for example, the charge on the whole phenolate ligand in 27-H,H,OMe,H and TS36-H,H,OMe,H are calculated as -0.640 and -0.192, respectively, implying that the phenolate in TS36-H,H,OMe,H has almost no phenoxenium character. A plausible explanation for this finding is that the hydrogen bond interaction of the carboxylate with the $\mathrm{OH}$ group in the transition structure increasingly enhances the electron density on the phenolate fragment, making the redox process independent of the substituent nature.

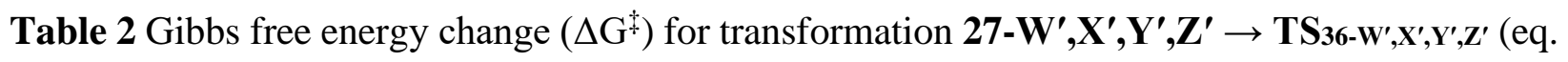
3) along with the potential energy change $(\Delta E)$ for the isodesmic reaction given in eq. 4 .<smiles>[Y]c1c([Y])c([Z])c(OI2OC(=O)c3ccccc32)c(O)c1[Y]</smiles>

27-'-', ,', , Y', $Z^{\prime}$

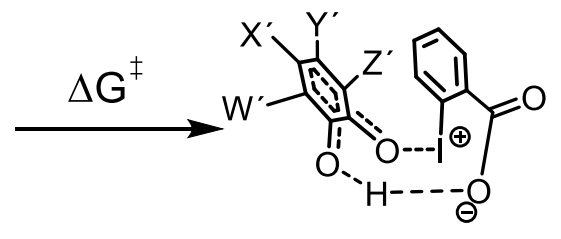

$\mathrm{TS}_{36}-\mathrm{W}^{\prime}, \mathrm{X}^{\prime}, \mathrm{Y}^{\prime}, \mathrm{Z}^{\prime}$

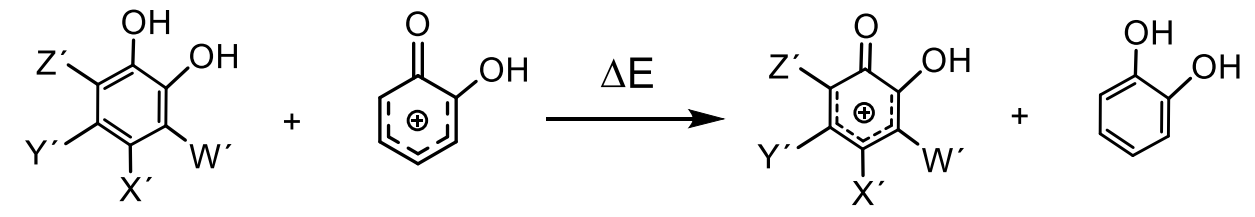

16-W','X', ,', Z'

$7^{\prime}$ $16^{\prime}$

\begin{tabular}{|c|c|c|c|c|c|}
\hline $\mathbf{W}^{\prime}$ & $\mathbf{X}^{\prime}$ & $\mathbf{Y}^{\prime}$ & $\mathbf{Z}^{\prime}$ & $\boldsymbol{\Delta} \mathbf{G}^{\star}$ & $\boldsymbol{\Delta}$ \\
\hline $\mathrm{H}$ & $\mathrm{H}$ & $\mathrm{H}$ & $\mathrm{H}$ & 22.2 & 0 \\
\hline $\mathrm{OMe}$ & $\mathrm{H}$ & $\mathrm{H}$ & $\mathrm{H}$ & 21.8 & 1.5 \\
\hline
\end{tabular}




\begin{tabular}{|c|c|c|c|c|c|}
\hline $\mathrm{H}$ & $\mathrm{H}$ & $\mathrm{OMe}$ & $\mathrm{H}$ & 22.8 & -1.5 \\
\hline $\mathrm{H}$ & $\mathrm{H}$ & $\mathrm{CN}$ & $\mathrm{H}$ & 20.5 & 11.1 \\
\hline $\mathrm{H}$ & $\mathrm{H}$ & $\mathrm{H}$ & $\mathrm{CN}$ & 23.5 & 8.8 \\
\hline
\end{tabular}

As a conclusion, we can say that the redox activation barrier with a transition structure having some phenoxenium character is lowered if the para position of the phenol is substituted by a strong electron donating group such as OMe. To further affirm this claim, we analysed the NPA charge on whole phenolate ligand in $\mathbf{T S}_{34}$ (pathway c in Fig. 7) and found that it has a partial charge of +0.458 . This result implies that the phenolate in $\mathbf{T S}_{34}$ features a significant phenoxenium character and thus the energy of such a transition structure should be lowered if the position of the OMe substituent is well tuned. To assess the validity of this statement, the iodine(III) reduction via pathway $c$ starting from $\mathbf{2 7 - H , O M e , H , H}$ were calculated (Fig. 10). In this phenolate complex, the OMe group is para to the I-O bond and so it should be capable of stabilizing the redox transition structure more effectively. Interestingly, consistent with our prediction, the redox reaction from 27-H,OMe,H,H via TS34' occurs with an activation free energy of $22.9 \mathrm{kcal} / \mathrm{mol}$, which is 8.6 $\mathrm{kcal} / \mathrm{mol}$ lower than that from 27-H,H,OMe,H via $\mathbf{T S}_{34}$ (Fig. 7).

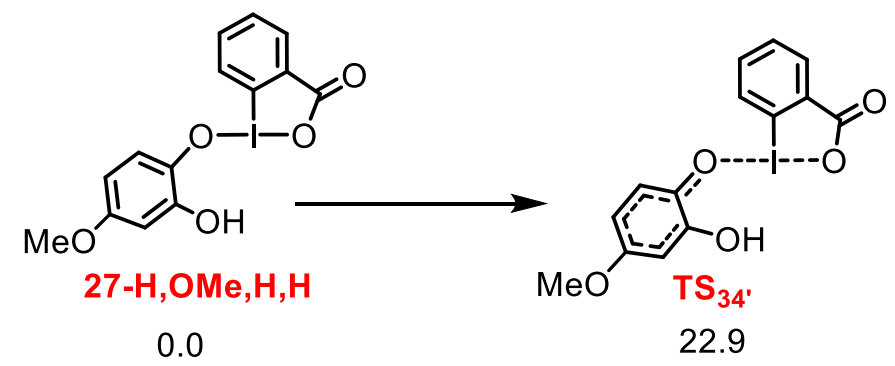

Fig. 10 Activation free energy for iodine(III) reduction starting from phenolate complex 27$\mathrm{H}, \mathrm{OMe}, \mathrm{H}, \mathrm{H}$ 


\section{Computational details}

Gaussian $16^{13}$ was used for the full optimization of all the structures involved in this work at the M06-2X level of theory. ${ }^{14,15}$ For all the optimised computations, the solvent effects were evaluated using the SMD solvation model with N,N'-dimethylformamide (DMF) as a solvent. ${ }^{16}$ The Hay and Wadt's effective core potential with a double- $\xi$ valence basis set (LANL2DZ) was used to describe iodine. ${ }^{17}$ For other atoms, the $6-31 \mathrm{G}(\mathrm{d})$ basis set was used. ${ }^{18}$ A polarization function was also added for $\mathrm{I}\left(\xi_{\mathrm{d}}=0.289\right) .{ }^{19}$ This basis set combination will be referred as BS1. Frequency calculations were also carried out at the M06-2X level of theory as used for the optimization of structures. Berny algorithm was used to locate all the transition structures. The connectivity between transition structures and minima was confirmed by intrinsic reaction coordinate (IRC) calculations. $^{20}$ The energies obtained from the SMD/M06-2X/LANL2DZ(d),6-31G(d) calculations were refined by single-point energy calculations with the M06-2X functional method for all the structures using a larger basis set (BS2) which employs the def2-TZVP basis set ${ }^{21}$ on all atoms. Effective core potentials including scalar relativistic effects were used for the iodine atom. To make the calculations more accurate, the tight convergence criterion was also involved. The free energy calculations for each species in solution was evaluated by using the following formula:

$\mathrm{G}=\mathrm{E}(\mathrm{BS} 2)+\mathrm{G}(\mathrm{BS} 1)-\mathrm{E}(\mathrm{BS} 1)+\Delta \mathrm{G}^{1 \mathrm{~atm} \rightarrow 1 \mathrm{M}}$

Where $\Delta \mathrm{G}^{1 \mathrm{~atm} \rightarrow 1 \mathrm{M}}=1.89 \mathrm{kcal} / \mathrm{mol}$ is the correction in free-energy change for compression of 1 mol of an ideal gas from 1 atm to the $1 \mathrm{M}$ solution phase standard state.

Minimum energy crossing point MECP1 between singlet intermediate 24 and triplet intermediate 33 (Fig. 4) were located using the code of Harvey et al. ${ }^{22}$ Our calculations showed that, in potential energy terms, MECP1 is about $5.2 \mathrm{kcal} / \mathrm{mol}$ higher in energy than $\mathbf{T S}$ 24-26-p-OMe. 


\section{Conclusion}

Mechanistic details of the phenol double oxidation mediated by IBX to yield an $o$ - quinone are provided in this study by using DFT calculations at the M06-2X level of theory. We found that the oxidation may proceed via four main steps: (i) the ligand exchange between the phenol substrate and IBX to give a phenolate complex, (ii) first redox reaction which installs an oxo group on the phenolate at its ortho position, (iii) tautomerisation which forms a catechol-iodine(III) complex (iv) second redox process which produces the final product concomitant with the reduction of iodine(III) to iodine(I). Our findings regarding this investigation are summarized as follows:

- The most stable isomer of IBX in which oxo ligand is trans to vacant coordination site is less likely to be involved in the ligand exchange and instead the process proceeds with a lower activation free energy if the IBX isomerizes to a less stable species with the oxo and carboxylate groups in a trans arrangement.

- The initial product of the ligand exchange process is an intermediate in which phenolate is trans to the vacant coordination site of IBX. The isomerization of this intermediate to a more stable form with the phenolate trans to the carboxylate ligand gives a species which is least reactive towards the first redox process.

- The less stable isomer of phenolate complex undergoes the redox process via an associative mechanism by which installation of oxo group on the phenolate and reduction of iodine(V) to iodine(III) occur in one step. The favourability of the associative mechanism over other variants reasonably explains the regioselectivity observed experimentally in the IBX-mediated oxidation of phenols.

- In contrast to the PIDA system, the phenol oxidation by IBX is unlikely to pass through an dearomatized phenolate intermediate. 
- The second redox process occurs from the catechol-iodine(III) complex via a carboxylateassisted transition structure wherein the iodine(III) reduction is facilitated by a hydrogen bond interaction between the catechol $\mathrm{OH}$ substituent and the carboxylate group.

- Although the iodine(V) reduction proceeds via an associative mechanism, its activation barrier shows a large dependence on the substituent in the phenol ring.

- An electron withdrawing substituent decreases the intrinsic stability of phenoxenium ion, whereas an electron donating substituent at ortho and especially para positions extremely increases it.

- There is a good correlation between the intrinsic stability of the phenoxenium and the activation barrier to the iodine $(\mathrm{V})$ reduction. This can be explained by the fact that the phenolate in the redox transition structure has some phenoxenium character.

- The presence of the hydrogen bond interaction between the catechol OH substituent and the carboxylate group in the transition structure relating to the iodine(III) reduction results in the energy barrier to the second redox process being mainly independent from the nature of the phenol substituent. Such an interaction causes the phenolate in the transition structure not to have any phenoxenium character.

- It is predicted that a redox activation barrier with a transition structure having some phenoxenium character is the lowest if the para position of the phenol is substituted by a strong electron donating group such as OMe.

\section{Notes and references}

1. For some selective reviews, see: (a) A. Yoshimura and V. V. Zhdankin, Advances in Synthetic Applications of Hypervalent Iodine Compounds, Chem. Rev., 2016, 116, 3328-3345; (b) Y. Li, D. 
P. Hari, M. V. Vita and J. Waser, Cyclic Hypervalent Iodine Reagents for Atom-Transfer Reactions: Beyond Trifluoromethylation, Angew. Chem., Int. Ed., 2016, 55, 4436-4454.

2. For some selective reviews regarding oxidative dearomatization of phenols, see: (a) A. M. Harned, Asymmetric oxidative dearomatizations promoted by hypervalent iodine(III) reagents: an opportunity for rational catalyst design?, Tetrahedron Lett., 2014, 55, 4681-4689; (b) S. Quideau, L. Pouysegu, P. A. Peixoto and D. Deffieux, Phenol Dearomatization with Hypervalent Iodine Reagents, Top. Curr. Chem., 2016, 373, 25-74; (c) W. T. Wu, L. Zhang and S. L. You, Catalytic asymmetric dearomatization (CADA) reactions of phenol and aniline derivatives, Chem. Soc. Rev., 2016, 45, 1570-1580; (d) L.Pouysegu, D. Deffieux and S. Quideau, Hypervalent iodine-mediated phenol dearomatization in natural product synthesis, Tetrahedron, 2010, 66, 2235-2261; (e) M. A. Ciufolini, N. A. Braun, S. Canesi, M. Ousmer, J. Chang and D. Chai, Oxidative amidation of phenols through the use of hypervalent iodine reagents: Development and applications, Synthesis, 2007, 3759-3772; (f) S. Quideau, L. Pouysegu and D. Deffieux, Oxidative Dearomatization of Phenols: Why, How and What For?, Synlett, 2008, 467-495; (g) H. Liang and M. A. Ciufolini, Angew. Chem., Int. Ed., 2011, 50, 11849-11851; (h) L. Pouysegu, T. Sylla, T. Garnier, L. B. Rojas, J. Charri, D. Deffieux and S. Quideau, Hypervalent iodine-mediated oxygenative phenol dearomatization reactions, Tetrahedron, 2010, 66, 5908-5917.

3. D. Magdziak, A. A. Rodriguez, R. W. Van De Water, and T. R. R. Pettus, Regioselective Oxidation of Phenols to o-Quinones with o-Iodoxybenzoic Acid (IBX), Org. Lett., 2002, 4, 285 288.

4. For some research articles regarding phenol oxidation by IBX, see: (a)L. Pouysegu, M. Marguerit, J. Gagnepain, G. Lyvinec, A. J. Eatherton and S. Quideau,Total synthesis of wasabidienones B1 and B0 via SIBX-mediated hydroxylative phenol dearomatization, Org. Lett., 2008, 10, 5211-5214;(b) Y. Huang, J. Zhang and T. R. R. Pettus, Synthesis of ( \pm )-Brazilin Using IBX, Org. Lett., 2005, 7, 5841-5844;(c) A. Boulange, P. A. Peixoto and X. Franck, Diastereoselective IBX Oxidative Dearomatization of Phenols by Remote Induction: Towards the Epicocconone Core Framewor, Chem. Eur. J. 2011, 17, 10241-10245; (d) A. Duschek and S. F. Kirsch, 2-Iodoxybenzoic Acid-A Simple Oxidant with a Dazzling Array of Potential Applications, Angew. Chem. Int. Ed., 2011, 50, 1524-1552; for computational studies see: (e) A. M. Harned, Concerning the mechanism of iodine(iii)-mediated oxidative dearomatization of phenols, Org. Biomol. Chem., 2018, 16, 2324-2329; (f) H. Zheng, Y. Sang, K. N. Houk, XiaoSong Xue, and Jin-Pie Cheng, Mechanism and Origins of Enantioselectivities in SpirobiindaneBased Hypervalent Iodine(III)-Induced Asymmetric Dearomatizing Spirolactonizations, J. Am. Chem. Soc. 2019, 141, 16046-16056.

5. B. Ganji and A. Ariafard, DFT mechanistic investigation into phenol dearomatization mediated by an iodine(III) reagent, Org. Biomol. Chem., 2019, 17, 3521-3528.

6. For some selective computational studies relating to organic transformations mediated by hypervalent iodine reagents, see: (a) K. Farshadfar, A. Chipman, B. F. Yates, A. Ariafard, DFT Mechanistic Investigation into $\mathrm{BF}_{3}$-Catalyzed Alcohol Oxidation by a Hypervalent Iodine(III) Compound, ACS Catal., 2019, 9, 6510-6521; (b) S. Izquierdo, S. Essafi, I. del Rosal, P. Vidossich, R. Pleixats, A. Vallribera, G. Ujaque, A. Lledos and A. Shafir, Acid activation in phenyliodine dicarboxylates: direct observation, structures, and implications, J. Am. Chem. Soc., 2016, 138, 1274712750; (c) A. Jobin-Des Lauriers and C. Y. Legault, Metathetical Redox Reaction of (Diacetoxyiodo)arenes and Iodoarenes, Molecules, 2015, 20, 22635-22644; (d) A. Sreenithya and R. B. Sunoj, Mechanistic Insights on Iodine(III) Promoted Metal-Free Dual C-H Activation Involved in the Formation of a Spirocyclic Bis-oxindole, Org. Lett., 2014, 16, 6224-6227; (e) C. Zhu, Y. Liang, X. 
Hong, H. Sun, W. Y. Sun, K. N. Houk and Z. Shi, Iodoarene-Catalyzed Stereospecific Intramolecular $\mathrm{sp}^{3}$ C-H Amination: Reaction Development and Mechanistic Insights, J. Am. Chem. Soc., 2015, 137, 7564-7567 ; (f) I. Funes-Ardoiz, W. M. C. Sameera, R. M. Romero, C. Martinez, J. A. Souto, D. Sampedro, K. Muniz and F. Maseras, DFT Rationalization of the Diverse Outcomes of the Iodine(III)Mediated Oxidative Amination of Alkenes, Chem.- Eur. J., 2016, 22, 7545-7553 ; (g) A. Sreenithya, K. Surya and R. B. Sunoj, Hypercoordinate iodine(III) promoted reactions and catalysis: an update on current mechanistic understanding, Wiley Interdiscip. Rev.: Comput. Mol. Sci., 2017, 7, e1299.

7. Recently, Tang and Harned reported that the oxidative dearomatization of phenols by PIDA is decelerated if the phenol is substituted by the electron-withdrawing groups. T. Tang and A. M. Harned, Experimental evidence for the formation of cationic intermediates during iodine(III)mediated oxidative dearomatization of phenols., Org. Biomol. Chem., 2018, 16, 8249-8252.

8. (a) J. N. Moorthy, K. Senapati and K. N. Parida, 6-Membered Pseudocyclic IBX Acids: Syntheses, X-ray Structural Characterizations, and Oxidation Reactivities in Common Organic Solvents, J. Org. Chem., 2010, 75, 8416-8421; (b) J. N. Moorthy, K. Senapati, K. N. Paridafor, S. Jhulki, K. Sooraj and N. N. Nair, Twist Does a Twist to the Reactivity: Stoichiometric and Catalytic Oxidations with Twisted Tetramethyl-IBX, J. Org. Chem., 2011, 76, 9593-9601; (c) D. B. Dess and J. C. Martin, A useful 12-I-5 triacetoxyperiodinane (the Dess-Martin periodinane) for the selective oxidation of primary or secondary alcohols and a variety of related 12-I-5 species, $J$. Am. Chem. Soc., 1991, 113, 7277-7287; (d) M. Frigerio, M. Santagostino and S. Sputore, A UserFriendly Entry to 2-Iodoxybenzoic Acid (IBX), J. Org. Chem., 1999, 64, 4537-4538; (e) J. T. Su and W. A. Goddard III, Enhancing 2-Iodoxybenzoic Acid Reactivity by Exploiting a Hypervalent Twist, J. Am. Chem. Soc., 2005, 127, 14146-14147.

9. Isomer $\mathbf{B}$ was also found to be responsible in driving the ligand exchange between amines and IBX. However, due to the lower acidity of amines with respect to phenols, pathway $b$ was calculated to be significantly favored over pathway $a$. A. Chipman, K. Farshadfar, J. A. Smith, B. F. Yates, A. Ariafard, DFT-Based Comparison between Mechanistic Aspects of Amine and Alcohol Oxidation Mediated by IBX, accepted for publication in J. Org. Chem.

10. The ligand exchange between IBX and phenol 16-p-OMe via transition structure $\mathbf{T S}_{\mathbf{2 2}} \mathbf{2}^{\prime} \mathbf{2} \mathbf{2}^{\prime}$ leads to intermediate $\mathbf{2 3}^{\prime}$ having the dearomatized phenolate trans to the $\mathrm{OH}$ ligand (Fig. 5). Since hydroxide is a stronger trans influencing ligand than acetate (the ligand in the PIDA system), formation of the dearomatized phenolate complex in the IBX coordination sphere becomes energetically less favorable, thereby results in the ligand exchange through this route being less likely to occur. To confirm this supposition, we replaced the $\mathrm{OH}$ ligand in $\mathbf{2 3}^{\prime}$ with OAc and found that the ensuing intermediate becomes significantly stable with a relative free energy of 13.8 $\mathrm{kcal} / \mathrm{mol}$ (Fig. S2).

11. An alternative for the second redox process is that the carboxylate ligand in intermediate $\mathbf{2 6}$ deprotonates the C-H bond via transition structure $\mathbf{T S}_{\mathbf{2 6 - 3 7}}$ (Fig S3) to give directly $\mathbf{3 7}$ and $\mathbf{3 8}$. Our calculation results indicate that this alternative with $\Delta \mathrm{G}^{\ddagger}=36.3 \mathrm{kcal} / \mathrm{mol}$ is unlikely to be competitive. For a similar scenario computationally investigated, see: (a) H. Jiang, T.-Y. Sun, Y. Chen, X. Zhang, Y.-D. Wu, Y. Xie and H. F. Schaefer III, Designing new Togni reagents by computation, Chem. Commun., 2019, 55, 5667-5670; (b) T.-Y. Sun, X. Wang, H. Geng, Y. Xie, Y.D. Wu, X. Zhang and H. F. Schaefer III, Why does Togni's reagent I exist in the high-energy hypervalent iodine form? Re-evaluation of benziodoxole based hypervalent iodine reagents, Chem. Commun., 2016, 52, 5371-5374. 
12) It was assumed that if phenol dearomatisation proceeds via an associative mechanism, then the activation barrier to the redox step is independent from the phenol substituent nature. For details, see ref 7.

13. M. J.Frisch, G. W. Trucks, H. B.Schlegel, G. E. Scuseria, M. A. Robb, J. R. Cheeseman, G.Scalmani, V. Barone, G. A. Petersson, H. Nakatsuji, X. Li, M. Caricato, A. V. Marenich, J. Bloino, B. G.Janesko, R.Gomperts, B.Mennucci, H. P.Hratchian, J. V.Ortiz, A. F.Izmaylov, J. L.Sonnenberg, D. Williams-Young, F. Ding, F. Lipparini, F. Egidi, J. Goings, B. Peng, A. Petrone, T. Henderson, D. Ranasinghe, V. G. Zakrzewski, J. Gao, N. Rega, G. Zheng, W. Liang, M. Hada, M. Ehara, K. Toyota, R. Fukuda, J. Hasegawa, M. Ishida, T. Nakajima, Y. Honda, O. Kitao, H. Nakai, T. Vreven, K. Throssell, J. A. Montgomery, J. E. Peralta, F. Ogliaro, M. J. Bearpark, J. J. Heyd, E. N. Brothers, K. N. Kudin, V. N. Staroverov, T. A. Keith, R. Kobayashi, J. Normand, K. Raghavachari, A. P. Rendell, J. C. Burant, S. S. Iyengar, J. Tomasi, M. Cossi, J. M. Millam, M. Klene, C. Adamo, R. Cammi, J. W. Ochterski, R. L. Martin, K. Morokuma, O. Farkas, J. B. Foresman and D. J. Fox, Gaussian 16, Revision A.03, Gaussian, Inc., Wallingford CT, 2016.

14. (a) Y. Zhao and D. G. Truhlar, The M06 suite of density functionals for main group thermochemistry, thermochemical kinetics, noncovalent interactions, excited states, and transition elements: two new functionals and systematic testing of four M06-class functionals and 12 other functionals, Theor. Chem. Acc., 2008, 120, 215-241; (b) Y. Zhao and D. G. Truhlar, Density Functionals with Broad Applicability in Chemistry, Acc. Chem. Res., 2008, 41, 157-167.

15. Recent benchmark calculations by Schaefer III et al. demonstrated that the M06-2X functional provides the most accurate energies for an organic reaction mediated by the hypervalent iodine oxidant. H. Jiang, Tian-Yu Sun, X. Wang, Y. Xie, X. Zhang, Yun-Dong Wu and H. F. Schaefer III, A Twist of the Twist Mechanism, 2-Iodoxybenzoic Acid (IBX)-Mediated Oxidation of Alcohol Revisited: Theory and Experiment, Org. Lett., 2017, 19, 6502-6505.

16. A. V. Marenich, C. J. Cramer and D. G. Truhlar, Universal Solvation Model Based on Solute Electron Density and on a Continuum Model of the Solvent Defined by the Bulk Dielectric Constant and Atomic Surface Tensions, J. Phys. Chem. B, 2009, 113, 6378-6396.

17. (a) P. J. Hay and W. R. Wadt, Ab initio effective core potentials for molecular calculations. Potentials for the transition metal atoms Sc to Hg, J. Chem. Phys., 1985, 82, 270-283; (b) W. R. Wadt and P. J. Hay, Ab initio effective core potentials for molecular calculations. Potentials for main group elements $\mathrm{Na}$ to $\mathrm{Bi}$, Chem. Phys., 1985, 82, 284-298.

18. P. C. Hariharan and J. A. Pople, The influence of polarization functions on molecular orbital hydrogenation energies, Theor. Chim. Acta, 1973, 28, 213-222.

19. A. Höllwarth, M. Böhme, S. Dapprich, A. W. Ehlers, A. Gobbi, V. Jonas, K. F. Köhler, R. Stegmann, A. Veldkamp and G. A. Frenking, A set of d-polarization functions for pseudo-potential basis sets of the main group elements $\mathrm{Al}, \mathrm{Bi}$ and f-type polarization functions for $\mathrm{Zn}, \mathrm{Cd}, \mathrm{Hg}$, Chem. Phys. Lett., 1993, 208, 237-240.

20. (a) K. Fukui, Formulation of the reaction coordinate, J. Phys. Chem., 1970, 74, 4161-4163; (b) K. Fukui, The path of chemical reactions - the IRC approach, Acc. Chem. Res., 1981, 14, 363-368.

21. F. Weigend, F. Furche and R. J. Ahlrichs, Gaussian basis sets of quadruple zeta valence quality for atoms H-Kr, Chem. Phys., 2003, 119, 12753-12762.

22. J. N. Harvey, M. Aschi, H. Schwarz and W. Koch, The singlet and triplet states of phenyl cation. A hybrid approach for locating minimum energy crossing points between non-interacting potential energy surfaces, Theor. Chem. Acc., 1998, 99, 95. 